\title{
Clinical implications of PTEN and VEGF expression status, as well as microvessel density in esophageal squamous cell carcinoma
}

\author{
WEI QU ${ }^{1,2^{*}}$, JIN-DONG FU $^{3 *}$, FANG YANG ${ }^{2}$, GONG-LI YANG $^{1}$, YA-LI ZHANG ${ }^{1}$, \\ XIN-YING WANG ${ }^{1}$, HONG-XIANG GU ${ }^{1}$, HAI-YAN ZHANG ${ }^{2}$ and LING WANG ${ }^{2}$
}

\author{
${ }^{1}$ Internal Medicine - Department of Digestive Diseases, Affiliated Hospital of Southern Medical University, \\ Guangzhou, Guangdong 510515; ${ }^{2}$ Internal Medicine - Department of Digestive Diseases, Rizhao People's Hospital, \\ Rizhao, Shandong 276800; ${ }^{3}$ Internal Medicine - Department of Digestive Diseases, \\ Shandong Medical University, Jinan, Shandong 250012, P.R. China
}

Received September 1, 2014; Accepted May 22, 2015

DOI: $10.3892 / 01.2015 .3431$

\begin{abstract}
There are limitations to the use of single biomarker levels, for example phosphate and tensin homology (PTEN) or vascular endothelial growth factor (VEGF), in the diagnosis of esophageal squamous cell carcinoma (ESCC). The present study therefore aimed to evaluate the clinical implications of combined detection of multiple biomarkers. The associations between PTEN and VEGF expression status, microvessel density (MVD), and the pathological characteristics of 50 patients with ESCC were determined using $\chi^{2}$, analysis of variance, and t-tests. The results indicated that the PTEN-positive rate was negatively correlated with ESCC histological grade $(\mathrm{P}<0.01)$, depth of ESCC invasion $(\mathrm{P}<0.01)$ and lymph node metastasis status. Furthermore, the VEGF-positive rate was correlated with lymph node metastasis status, while MVD was correlated with the depth of ESCC invasion $(\mathrm{P}<0.01)$ and lymph node metastasis status $(\mathrm{P}<0.05)$. The PTEN-positive rate was negatively correlated with the VEGF-positive rate. A higher MVD was identified in ESCC samples than that of the normal esophageal mucosa, particularly in VEGF-positive ESCC specimens compared with those of VEGF-negative specimens, and PTEN-negative ESCC specimens compared with that of the PTEN-positive ESCC specimens. These results suggested that combined detection of PTEN and VEGF levels, as well as evaluation of MVD in patients with ESCC
\end{abstract}

Correspondence to: Dr Ya-Li Zhang, Internal Medicine - Division of Digestive Diseases, Affiliated Hospital of Southern Medical University, 1838 North Guangzhou Avenue, Guangzhou, Guangdong 510515, P.R. China

E-mail: zyl41531@163.com; quwei604@163.com

${ }^{*}$ Contributed equally

Key words: esophageal squamous cell carcinoma, phosphate and tensin homolgy, vascular endothelial growth factor, microvessel density, cluster of differentiation 31 may provide essential information for improvements in the diagnosis and prognosis of ESCC.

\section{Introduction}

Esophageal squamous cell carcinoma (ESCC) is ranked as the sixth leading cause of cancer-associated mortality worldwide, and is prevalent and particularly common in Asia (1). Although significant progress has been made in the development of surgical and adjuvant chemoradiotherapeutic techniques, the diagnosis and prognosis of patients with ESCC has remained poor. Investigations into alterations in the levels of specific proteins that occur in this cancer may provide clues to indicate novel biomarkers, required in order to improve diagnosis and guide targeted therapy.

Angiogenesis is required for tumor growth, and can be quantified as the microvessel density (MVD), which has become the morphological gold standard used to assess the neovasculature of human tumors (2). Newly formed microvessels are able to be visualized by cluster of differentiation 31 (CD31) staining in endothelial cells (3). However, there are disadvantages to this technique of angiogenesis detection, including potential cross-reactions with plasma cells and loss of antigens due to fixatives. Results using anti-CD31 antibody alone may erroneously indicate MVD in the diagnosis and prognosis of human tumors (2).

Vascular endothelial growth factor (VEGF) is an angiogenic factor that promotes the proliferation and migration of endothelial cells, enhances the permeability of blood vessels, reduces apoptosis of endothelial cells and promotes stromal proteolysis (4). VEGF therefore has a critical role in angiogenesis in numerous solid malignancies. The effect of VEGF on progression and recurrence of esophageal cancer has previously been investigated (5); however, the association between VEGF overexpression and the prognosis of patients with ESCC remains controversial.

Phosphate and tensin homology (PTEN) is a phosphatase with dual-specificity for proteins and lipids, which inhibits cell migration, spreading and focal adhesions (6). Studies of certain cancer cell lines have suggested that PTEN dysregulation may be involved in almost all types of cancer, including solid 
tumors and hematological malignancies (7-9). A study revealed that decreased levels of PTEN were correlated with tumor differentiation, infiltration depth and pTNM staging (10). The 5-year survival rate of patients with PTEN-positive ESCC was significantly higher than that of patients with PTEN-negative ESCC (10). This suggested that PTEN may have a significant role in carcinogenesis and the progression of ESCC, and therefore, may have significant clinical implications.

Analysis of a single protein biomarker is insufficient for accurate prediction of clinical outcome. CD31, PTEN and VEGF are functionally distinct molecules and may underlie various mechanisms in the pathogenesis and progression of specific types of cancer. The objective of the present study was therefore to investigate the clinical implications of PTEN and VEGF expression status and MVD, as well as their associations in ESCC, in order to provide insight into the development of techniques for the diagnosis and prognosis of ESCC. The PTEN and VEGF levels and MVD were determined in ESCC tumor and normal specimens from patients using immunohistological staining.

\section{Materials and methods}

Patients and specimens. The Institutional Review Board of Rizhao People's Hospital (Rizhao, China) approved the present study, and all participants provided written informed consent.

The study included 50 patients with ESCC (31 male, 19 female; aged $57.45 \pm 7.53$ years; age range, $30-75$ years) who underwent esophagectomy at the First Affiliated Hospital of Southern Medical University (Guangzhou, China) or Rizhao People's Hospital between January 2009 and January 2014. None of the patients had received radiotherapy or chemotherapy prior to surgery. ESCC specimens and adjacent normal mucosa were collected via gastrointestinal endoscopic biopsy.

ESCC specimens were confirmed by tumor histopathology, with screening and evaluation conducted by two experienced pathologists blinded to the clinical information. Normal specimens were collected from the distal normal mucosa. Within 30 min of surgery, each specimen was divided into two sections: One was flash-frozen in liquid nitrogen and stored at $-80^{\circ} \mathrm{C}$, while the other was stored in $4 \%$ buffered formamide (Laiyang Fine Chemical Factory, Laiyang, China). Specimens stored in $4 \%$ buffered formamide were used for standard clinicopathological diagnosis and immunohistological staining.

Immunohistological staining. All materials were purchased from Fuzhou Maixin Biotechnology Development Co., Ltd. (Fuzhou, China), unless otherwise stated. The PTEN and VEGF levels, as well as the presence of CD31 was detected by immunohistochemistry using an Ultrasensitive $\mathrm{S}-\mathrm{P}$ kit, in accordance with the manufacturer's instructions. Briefly, specimen slices were dewaxed and then incubated in $3 \%$ hydrogen peroxide for $30 \mathrm{~min}$ at room temperature to block endogenous peroxidase activity. Following rinsing with phosphate-buffered saline (PBS; batch no. 14122303) and incubation in citrate buffer $(10 \mathrm{mM}, \mathrm{pH} 6.0$; batch no. 14073001$)$ at $95^{\circ} \mathrm{C}$ for $20 \mathrm{~min}$, slices were blocked with rabbit serum for $30 \mathrm{~min}$. The slices were then incubated with biotin-conjugated primary antibodies [monoclonal anti-PTEN (cat. no. MAB-0369), anti-VEGF (cat. no. MAB-0243) or
anti-CD31 (cat. no. MAB-0031), all 1:100) at $4^{\circ} \mathrm{C}$ overnight, prior to incubation with horseradish peroxidase-labeled streptavidin $(1: 50)$ at $37^{\circ} \mathrm{C}$ for $30 \mathrm{~min}$. After rinsing with PBS, diaminobenzidine (batch no. 1409030031) was added and the sections were washed with tap water. Subsequently, the slices were processed sequentially with hematoxylin (Shanghai Ruji Biotechnology Development Co., Ltd., Shanghai, China), alcohol dehydration and xylene (Shanghai Aibi Chemistry Preparation Co., Ltd, Shanghai, China). Finally, the slices were mounted on slides and sealed with neutral balsam resin (Genetech Co., Ltd., Shanghai, China).

Breast cancer tissues known to express PTEN and colon cancer tissues known to express VEGF were prepared by histologists in the Department of Histology, Rizhao People's Hospital. These were used as references for PTEN and VEGF expression, respectively.

Determination of PTEN-and VEGF-positivity, and MVD. Following immunohistological staining with anti-PTEN antibody, brownish-yellow or light yellow granules in the cytoplasm or nuclei of cells were considered to indicate PTEN expression. Using an Olympus CH30 microscope (Olympus Corporation, Tokyo, Japan), ten microscopic fields (magnification, x400) were randomly selected and $>1,000$ cells were counted for each specimen. If $\leq 10 \%$ of cells expressed PTEN, the specimen was scored as PTEN-negative. If 11-100\% of the cells expressed PTEN, the specimen was scored as PTEN-positive.

For specimens stained with anti-VEGF antibody, brownish-yellow granules in the cytoplasm or cytoplasmic membrane of cells were considered indicative of VEGF expression. If $\leq 50 \%$ of cells contained VEGF, the specimen was scored as VEGF-negative, whereas if $51-100 \%$ of cells contained VEGF, the specimen was scored as VEGF-positive.

MVD was determined in accordance with a previously published method (4). The microvessels were identified by immunohistological staining for CD31. The number of microvessels was counted in three randomly selected microscopic fields in the vascular areas (magnification, x400), and the mean was calculated.

Statistical analysis. The $\chi^{2}$ test, one-way analysis of variance and Student's t-test were used to evaluate data. $\mathrm{P}<0.05$ was considered to indicate a statistically significant difference for all tests. All statistical analyses were performed using SPSS 18.0 (SPSS, Inc., Chicago, IL, USA).

\section{Results}

Histopathological characteristics of ESCC. The ESCC tumors were classified based on pathological characteristics. According to the differentiation status (11), each ESCC specimen was assigned one of three grades: 19 cases were well differentiated (Grade I), 17 cases were moderately differentiated (Grade II) and 14 cases were poorly differentiated (Grade III). ESCC specimens were also divided by depth of invasion: 15 cases were classified as level I, in which the tumor was found in the mucosa, submucosa or superficial muscle; while 35 cases were level II, in which the tumor was in the deep muscular or outer layer. A total of 19 cases were positive for metastasis, where tumors were identified in the esophageal lymph nodes 
Table I. Association between PTEN status, VEGF status, MVD and ESCC.

A, PTEN expression

\begin{tabular}{lcccccc}
\hline Specimen type & Cases, $\mathrm{n}$ & Negative, $\mathrm{n}$ & Positive, $\mathrm{n}$ & Positive rate, $\%$ & $\chi^{2}$ & P-value \\
\hline Normal & 50 & 0 & 50 & 100 & 27.8701 & $<0.0000$ \\
ESCC & 50 & 23 & 27 & 54 & \\
\hline
\end{tabular}

B, VEGF expression

\begin{tabular}{lccccc}
\hline Specimen type & Cases, $n$ & Negative, $n$ & Positive, $n$ & Positive rate, $\%$ & $\chi^{2}$ \\
\hline Normal & 45 & 45 & 0 & 0 & 47.6557 \\
ESCC & 50 & 16 & 34 & 68 & $<0.0000$ \\
\hline
\end{tabular}

C, MVD

\begin{tabular}{|c|c|c|c|c|}
\hline Specimen type & Cases, $\mathrm{n}$ & MVD/microscopic field, mean \pm SD & t-value ${ }^{a}$ & P-value \\
\hline Normal & 47 & $22.75 \pm 8.82$ & 8.2205 & $<0.0000$ \\
\hline ESCC & 50 & $37.82 \pm 9.21$ & & \\
\hline
\end{tabular}

$\chi^{2}$ values indicate the correlation between positive and negative expression for specimen type; $t$-values indicate the association between the specimen type and MVD/microscopic field. aDetermined by performing Student's t-test. PTEN, phosphate and tensin homology; VEGF, vascular and endothelial growth factor; MVD, microvascular density; ESCC, esophageal squamous cell carcinoma; SD, standard deviation.
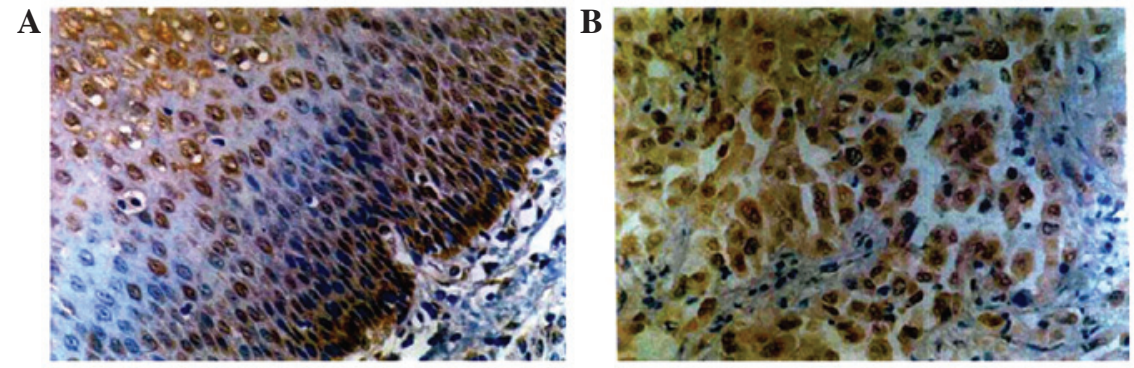

Figure 1. Expression status of phosphate and tensin homolog in (A) normal specimens and (B) esophageal tumor (magnification, $\mathrm{x} 400$ ).
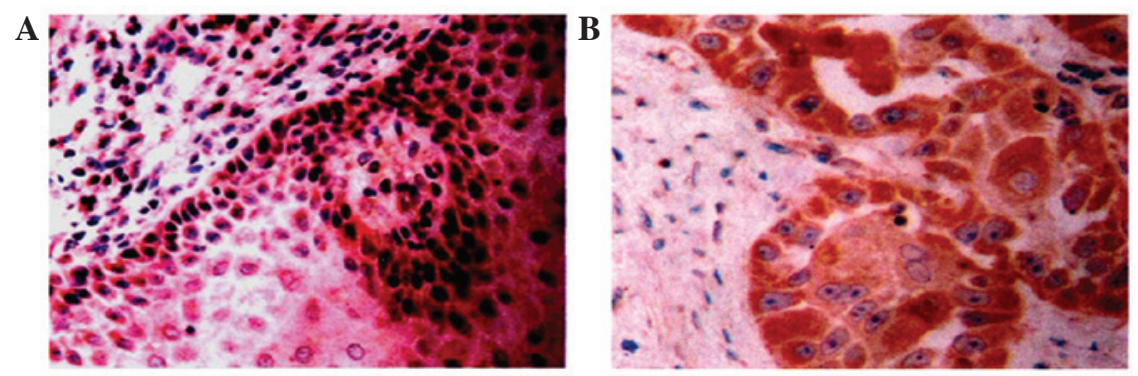

Figure 2. Expression status of vascular endothelial growth factor in (A) normal specimens and (B) esophageal tumor (magnification, x400).

of the ESCC specimens. No tumors were detected in the esophageal lymph nodes of the remaining 31 specimens (the metastasis-negative group).

MVD and PTEN/VEGF expression in ESCC and normal specimens. To determine differences in the MVD, as well as PTEN and VEGF expression status between ESCC and normal tissue specimens, an immunohistological staining assay using anti-CD31, anti-PTEN and anti-VEGF antibodies, respectively, was performed (Figs. 1-3).

The positive or negative status of PTEN and VEGF expression in the specimens was determined by the percentage of 
Table II. Associations between PTEN status, VEGF status and MVD, and histological characteristics of ESCC.

A, PTEN expression

\begin{tabular}{|c|c|c|c|c|c|c|}
\hline Histological characteristic & Cases, $\mathrm{n}$ & PTEN - & PTEN + & Positive rate, $\%$ & $\chi^{2}$ & P-value \\
\hline \multicolumn{7}{|l|}{ Differentiation grade } \\
\hline $\mathrm{I}$ & 19 & 4 & 15 & 78.9 & 11.4693 & 0.0032 \\
\hline II & 17 & 10 & 7 & 41.2 & & \\
\hline III & 14 & 11 & 3 & 21.4 & & \\
\hline \multicolumn{7}{|l|}{ Depth of invasion } \\
\hline Level I & 15 & 3 & 12 & 80.0 & 6.7308 & 0.0095 \\
\hline Level II & 35 & 21 & 14 & 40.0 & & \\
\hline \multicolumn{7}{|l|}{ Lymph node metastasis } \\
\hline Positive & 19 & 13 & 10 & 31.6 & 6.2019 & 0.0128 \\
\hline Negative & 31 & 6 & 21 & 67.7 & & \\
\hline
\end{tabular}

$\mathrm{B}, \mathrm{VEGF}$ expression

\begin{tabular}{|c|c|c|c|c|c|c|}
\hline Histological characteristic & Cases, $\mathrm{n}$ & VEGF - & $\mathrm{VEGF}+$ & Positive rate, $\%$ & $\chi^{2}$ & P-value \\
\hline \multicolumn{7}{|l|}{ Differentiation grade } \\
\hline I & 19 & 9 & 10 & 52.6 & 2.3596 & 0.3073 \\
\hline II & 17 & 6 & 11 & 64.7 & & \\
\hline III & 14 & 3 & 11 & 78.6 & & \\
\hline \multicolumn{7}{|l|}{ Depth of invasion } \\
\hline Level I & 15 & 7 & 8 & 53.3 & 1.0582 & 0.3036 \\
\hline Level II & 35 & 11 & 24 & 68.6 & & \\
\hline \multicolumn{7}{|l|}{ Lymph node metastasis } \\
\hline Positive & 19 & 3 & 16 & 84.2 & 5.4329 & 0.0198 \\
\hline Negative & 31 & 15 & 16 & 51.6 & & \\
\hline
\end{tabular}

C, MVD

\begin{tabular}{|c|c|c|c|c|}
\hline Histological characteristic & Cases, $\mathrm{n}$ & MVD/microscopic field, mean \pm SD & F-value ${ }^{a}$ & P-value \\
\hline \multicolumn{5}{|l|}{ Differentiation grade } \\
\hline I & 19 & $32.53 \pm 9.64$ & 2.6800 & 0.0788 \\
\hline II & 17 & $35.35 \pm 8.75$ & & \\
\hline III & 14 & $40.26 \pm 10.16$ & & \\
\hline \multicolumn{5}{|l|}{ Depth of invasion } \\
\hline Level I & 15 & $30.52 \pm 8.54$ & 3.8255 & 0.0004 \\
\hline Level II & 35 & $42.35 \pm 10.57$ & & \\
\hline \multicolumn{5}{|l|}{ Lymph node metastasis } \\
\hline Positive & 19 & $46.57 \pm 5.62$ & 7.3763 & $<0.0000$ \\
\hline Negative & 31 & $35.76 \pm 4.64$ & & \\
\hline
\end{tabular}

$\chi^{2}$ values indicate correlation between positive and negative expression for each characteristic; $t$-values indicate the association between each histological characteristic and MVD/miscroscopic field. ${ }^{\mathrm{a} F}$-values were determined by one-way analysis of variance. PTEN, phosphate and tensin homology; VEGF, vascular endothlial growth factor; MVD, microvascular density.

cells that were positively stained (Table I). Of the 50 ESCC specimens, 27 and 23 cases were PTEN-positive and -negative, respectively, while all normal healthy specimens were PTEN-positive.
A total of 34 cases of ESCC were VEGF-positive, while none of the normal esophageal mucosa samples were VEGF-positive. The MVD was significantly higher in ESCC tissues (37.82 $\pm 9.21 /$ microscopic field) than that of the normal 

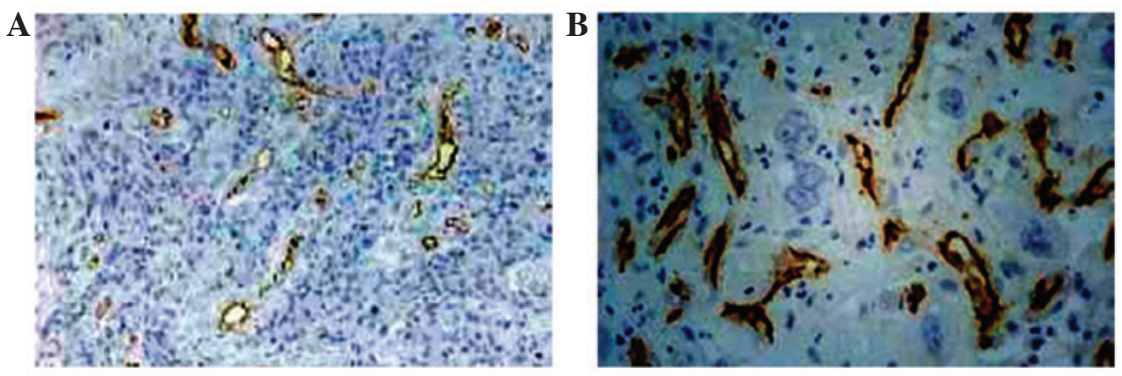

Figure 3. Microvascular density in esophageal squamous cell carcinoma at (A) magnification, x100 and (B) x400.

esophageal mucosa $(22.75 \pm 8.82 /$ microscopic field; $\mathrm{P}<0.01)$. These results suggested that the expression of PTEN was significantly decreased, while VEGF expression and MVD were significantly increased in ESCC, compared with that of the normal specimens. Therefore, PTEN and VEGF levels and the MVD may be clinically implicated in ESCC.

To determine whether PTEN and VEGF levels and MVD were associated with ESCC, a $\chi^{2}$ test was performed. The results indicated that PTEN-negative status, VEGF-positive status and greater MVD were significantly correlated with ESCC (Table I). These results suggested that a low percentage of cells staining positive for PTEN, a high percentage staining positive for VEGF and high MVD may be used in the diagnosis of ESCC.

Association between PTEN status, VEGF status, MVD and clinicopathological characteristics of ESCC. To determine whether PTEN and VEGF levels and MVD were associated with histological grades of ESCC, a $\chi^{2}$ test was used to examine the correlation between PTEN and VEGF expression status, the MVD, and the differentiation status of ESCC. PTEN-positive status was found to be negatively correlated with the histological grade of ESCC $(\mathrm{P}<0.01)$, while VEGF-positive status and MVD were not correlated with the histological grade of ESCC (P>0.05; Table II). The results suggested that loss of PTEN expression may be an indicator of differentiation status, carcinogenesis and progression of ESCC.

To determine whether levels of PTEN and VEGF and MVD may be used to predict the depth of ESCC invasion, a $\chi^{2}$ test was performed to examine correlation between the levels of PTEN and VEGF, and MVD, and the depth of ESCC invasion (Table II). The results indicated that a PTEN-positive status was negatively correlated with depth of ESCC invasion $(\mathrm{P}<0.01)$, while VEGF-positive status exhibited no correlation and MVD was positively correlated with depth of ESCC invasion $(\mathrm{P}<0.001)$. These results suggested that decreased PTEN expression and increased MVD, but not VEGF, were indicators of ESCC invasion.

To determine whether PTEN status, VEGF status, and MVD were viable indicators for predicting metastasis of ESCC, a $\chi^{2}$ test was used to examine the correlation between PTEN status, VEGF status and MVD, and the lymph node metastasis status of ESCC. The results indicated that PTEN-negative and VEGF-positive status, and higher MVD were correlated with lymph node metastasis of ESCC specimens $(\mathrm{P}<0.05$; Table II). These results suggested that
Table III. Association between PTEN and VEGF status.

\begin{tabular}{lrrrr}
\hline & \multicolumn{2}{c}{ VEGF } & & \\
\cline { 2 - 3 } & + & - & $\chi^{2}$ & P-value \\
\hline PTEN & 12 & 15 & 14.9670 & 0.0001 \\
+ & 22 & 1 & & \\
- & & & & \\
\hline
\end{tabular}

PTEN, phosphate and tensin homology; VEGF, vascular and endothelial growth factor; MVD.

Table IV. Association of PTEN-negative status and VEGF-positive status with high MVD.

\begin{tabular}{lccc}
\hline & MVD & t-value & P-value \\
\hline PTEN & & & \\
+ & $33.58 \pm 8.92$ & 3.3145 & 0.0018 \\
- & $42.39 \pm 9.87$ & & \\
VEGF & & & \\
+ & $42.53 \pm 8.57$ & 4.1084 & 0.0002 \\
- & $32.25 \pm 7.51$ & & \\
\hline
\end{tabular}

PTEN, phosphate and tensin homology; VEGF, vascular and endothelial growth factor; MVD, microvascular density.

decreased PTEN levels and increased VEGF and MVD were indicators of metastasis in ESCC.

Association between PTEN status, VEGF status and MVD in ESCC. To determine whether PTEN levels were associated with VEGF levels in ESCC, a $\chi^{2}$ test was performed (Table III). The results revealed that the PTEN expression profile was negatively correlated with the VEGF expression profile $(\mathrm{P}<0.01$; Table III). Subsequently, to determine whether MVD was associated with the levels of PTEN and VEGF in ESCC, a t-test was conducted. The results indicated that the MVD was significantly lower in PTEN-positive ESCC specimens than in PTEN-negative ESCC specimens $(\mathrm{P}=0.0018)$, while MVD was significantly higher in VEGF-positive ESCC specimens than in VEGF-negative ESCC specimens $(\mathrm{P}=0.0002$; Table IV). These results suggested that decreased 
PTEN expression, increased VEGF expression, and high MVD are associated in ESCC.

\section{Discussion}

The analysis of a single protein biomarker is insufficient for adequate and accurate diagnosis and prognosis of ESCC. However, the examination of multiple biomarker proteins may provide more accurate information. In the current study, the expression profiles of PTEN and VEGF, and the MVD were investigated in ESCC and normal specimens. Of MVD and expression statuses of PTEN and VEGF, only PTEN expression was found to negatively correlate with the histological grade of ESCC. PTEN expression and MVD were correlated with the depth of ESCC invasion, while PTEN expression, VEGF expression and MVD were correlated with the lymph node metastasis status of ESCC specimens. Furthermore, it was demonstrated that the expression of PTEN was negatively correlated with the expression of VEGF, while a higher MVD was associated with higher VEGF and lower PTEN expression. Therefore, it was concluded that examination of MVD and the expression status of PTEN and VEGF may provide improved information for the diagnosis and prognosis of ESCC.

Angiogenesis is required for tumor growth and metastasis (12), and may be evaluated by staining endothelial cell markers CD31 and CD34 $(13,14)$. This method has previously been applied for the determination of the role of the neovasculature in extrahepatic cholangiocarcinoma (4) and Dukes' B colon cancer (15). These studies demonstrate the significant effects of vascularization on the survival of patients with extrahepatic cholangiocarcinoma and Dukes B colon cancer. In the current study, it was revealed that MVD was correlated with the depth of invasion and lymph node metastasis of ESCC. Therefore, determination of MVD may facilitate prediction of the depth of invasion and lymph node metastasis of ESCC.

VEGF is an angiogenic factor, which promotes proliferation and migration of endothelial cells, enhances permeability of blood vessels, reduces apoptosis of endothelial cells and increases stromal proteolysis (16). Studies have indicated that VEGF has a crucial role in angiogenesis in various solid malignancies. VEGF-C is lymphangiogenic, and is able to selectively induce hyperplasia of the lymphatic vasculature. VEGF-C expression is correlated with lymph node metastasis and poor prognosis in esophageal cancer (17-19). Furthermore, in patients with esophageal tumors, the expression of VEGF-C has been shown to be an effective diagnostic factor for determining metastasis of lymph nodes (17-19). In a meta-analysis, elevated VEGF expression was shown to be associated with poor survival in patients with esophageal cancer (20). Inhibition of VEGF-induced angiogenesis suppresses tumor growth in vivo (21). The results of these studies support the hypothesis of a role for VEGF expression as an indicator for predicting poor survival in patients with esophageal carcinoma, and may have implications for treatments directed at inhibiting VEGF-mediated angiogenesis. In the current study, it was demonstrated that upregulation of VEGF protein expression was associated with increased MVD in ESCC. Furthermore, VEGF expression was correlated with lymph node metastasis, but not with differentiation status or depth of invasion. Therefore, VEGF expression may be a predictor of risk for lymph node metastasis in esophageal cancer.

PTEN is a phosphatase with dual-specificity for protein and lipids. The PTEN gene has an important role in cell proliferation, differentiation and apoptosis. Studies conducted in certain cancer cell lines indicated that the loss of PTEN expression may underlie almost all types of cancer, including solid tumors and hematological malignancies. For example, the loss of PTEN promotes tumorigenesis of breast tumors (22). In the present study, decreased PTEN expression was found to be correlated with differentiation status of ESCC. This is consistent with the results of several previous studies (6-8). Therefore, PTEN is likely involved in the tumorigenesis and progression of ESCC.

In addition to correlation with differentiation status, decreased PTEN expression was also correlated with depth of invasion and lymph node metastasis of ESCC. Studies have previously suggested that PTEN may regulate interactions between cells and the extracellular matrix by interacting with focal adhesion kinase (FAK) and thereby reducing tyrosine phosphorylation. Therefore, PTEN inhibits cell migration, spreading and focal adhesion. Reduced PTEN expression may regulate integrin-mediated adhesion through dephosphorylation of FAK and increased cell spreading (5). PTEN may suppress cell adhesion to the extracellular matrix through its serine/threonine phosphatase role, for example, as a negative regulator of the formation of stable HT-29 cell adhesion to the extracellular matrix (23). Decreased PTEN expression has roles in the tumorigenesis, progression, growth, differentiation and angiogenesis of gastric cancer, likely via inhibition of the expression of caspase-3 in tumor cells (24). In prostate basal cells, conditionally-ablated PTEN enhances basal-to-luminal differentiation and induces invasive prostate cancer in mice (25). Hypermethylation of PTEN may inhibit the expression of PTEN, and PTEN hypermethylation was found to be associated with ESCC in Chinese Kazakh patients (26). Therefore, PTEN may be involved in the regulation of cell growth, tumor invasion, metastasis and angiogenesis.

The regulation of PTEN and VEGF expression in ESCC appears to occur via two distinct processes. However, these factors exert opposite effects on angiogenesis in tumors. PTEN functions as a tumor suppressor gene and inhibits the formation of blood vessels in tumors, while VEGF promotes tumor vascularization. Akt is a serine/threonine kinase involved in cell growth and survival. Activation of Akt requires phosphatidylinositol $(3,4,5)$-triphosphate (PIP3), a second messenger in cells. PTEN dephosphorylates PIP3, thereby functioning in opposition to phosphatidylinositol 3-kinase (PI3K), which has a crucial role in the formation of PIP3. By blocking Akt activation, PTEN regulates multiple cellular processes, including cell cycling, translation and apoptosis (6). In addition, PI3K has a key role in angiogenesis and regulates VEGF expression. Overexpression of PTEN or of dominant-negative constructs of PI3K attenuates angiogenesis in the yolk sac of chicken embryos, indicating that PI3K and Akt signaling is required for normal embryonal angiogenesis (27). PTEN and VEGF function in the PI3K-PIP3-AKT network and are key regulators of cell growth in multiple pathways (28). Studies have demonstrated that inactivation of the PTEN gene and 
overexpression of $V E G F$, contribute to the neovascularisation and progression of gastric cancer (29). Angiogenesis due to decreased PTEN expression may be attributed to the corresponding upregulation of VEGF levels. In the current study, it was demonstrated that a reduction or loss of PTEN expression was correlated with elevated VEGF protein levels in ESCC. Therefore, PTEN and VEGF are interrelated indicators of ESCC. The specific mechanisms underlying PTEN and VEGF interaction remain to be elucidated.

In conclusion, PTEN/VEGF expression status and MVD are differentially associated with characteristics of ESCC. The differentiation status of ESCC may be determined by decreased PTEN expression, while invasion of ESCC is likely associated with decreased PTEN expression levels and higher MVD. Metastasis of ESCC is associated with decreased PTEN expression levels, enhanced VEGF expression levels and higher MVD. Combined detection of PTEN and VEGF expression levels and MVD may provide information essential for improvements in the diagnosis and prognosis of ESCC.

\section{Acknowledgements}

The present study was financially supported by the Affiliated Hospital of Southern Medical University (Guangzhou, China), (grant no. 2012B050600020).

\section{References}

1. Ferlay J, Shin HR, Bray F, Forman D, Mathers C and Parkin DM: Estimates of worldwide burden of cancer in 2008: GLOBOCAN 2008. Int J Cancer 127: 2893-2917, 2010.

2. Nico B, Benagiano V, Mangieri D, Maruotti N, Vacca A and Ribatti D: Evaluation of microvascular density in tumors: Pro and contra. Histol Histopathol 23: 601-607, 2008.

3. Torres C, Wang H, Turner J, Shahsafaei A and Odze RD: Prognostic significance and effect of chemoradiotherapy on microvessel density (angiogenesis) in esophageal Barrett's esophagus-associated adenocarcinoma and squamous cell carcinoma. Hum Pathol 30: 753-758, 1999.

4. Möbius C, Demuth C, Aigner T, Wiedmann M, Wittekind C, Mössner J, Hauss J and Witzigmann H: Evaluation of VEGF A expression and microvascular density as prognostic factors in extrahepatic cholangiocarcinoma. Eur J Surg Oncol 33 : 1025-1029, 2007

5. Kleespies A, Guba M, Jauch KW and Bruns CJ: Vascular endothelial growth factor in esophageal cancer. J Surg Oncol 87 95-104, 2004.

6. Tamura M, Gu J,Matsumoto K, Aota S,Parsons R and Yamada KM: Inhibition of cell migration, spreading and focal adhesions by tumor suppressor PTEN. Science 280: 1614-1617, 1998.

7. Simpson L and Parsons R: PTEN: Life as a tumor suppressor. Exp Cell Res 264: 29-41, 2001.

8. Dahia PL: PTEN, a unique tumor suppressor gene. Endocr Relat Cancer 7: 115-129, 2000.

9. Chu EC and Tarnawski AS: PTEN regulatory functions in tumor suppression and cell biology. Med Sci Monit 10: RA235-RA241, 2004.

10. Chang D, Wang TY, Li HC, Wei JC and Song JX: Prognostic significance of PTEN expression in esophageal squamous cell carcinoma from Linzhou City, a high incidence area of northern China. Dis Esophagus 20: 491-496, 2007.
11. Petersen I: The new WHO classification and recent results in soft tissue tumor pathology. Pathologe 34: 436-448, 2013 (In German).

12. Blood $\mathrm{CH}$ and Zetter BR: Tumor interactions with the vasculature: Angiogenesis and tumor metastasis. Biochim Biophys Acta 1032: 89-118, 1990.

13. Weidner N: Chapter 14. Measuring intratumoral microvessel density. Methods Enzymol 444: 305-323, 2008.

14. Weidner N: Intratumor microvessel density as a prognostic factor in cancer. Am J Pathol 147: 9-19, 1995.

15. Sundov Z, Tomic S, Alfirevic S, Sundov A, Capkun V, Nincevic Z, Nincevic J, Kunac N, Kontic M, Poljak N, et al: Prognostic value of MVD, LVD and vascular invasion in lymph node-negative colon cancer. Hepatogastroenterology 60: 432-438, 2013.

16. Hoeben A, Landuyt B, Highley MS, Wildiers H, Van Oosterom AT and De Bruijn EA: Vascular endothelial growth factor and angiogenesis. Pharmacol Rev 56: 549-580, 2004.

17. Tanaka T, Ishiguro H, Kuwabara Y, Kimura M, Mitsui A, Katada T, Shiozaki M, Naganawa Y, Fujii Y and Takeyama H: Vascular endothelial growth factor C (VEGF-C) in esophageal cancer correlates with lymph node metastasis and poor patient prognosis. J Exp Clin Cancer Res 29: 83, 2010.

18. Kozlowski M, Naumnik W, Niklinski J, Milewski R, Dziegielewski P and Laudanski J: Vascular endothelial growth factor $C$ and $D$ expression correlates with lymph node metastasis and poor prognosis in patients with resected esophageal cancer. Neoplasma 58: 311-319, 2011.

19. Okazawa T, Yoshida T, Shirai Y, Shiraishi R, Harada T, Sakaida I, Abe T and Oka M: Expression of vascular endothelial growth factor $\mathrm{C}$ is a prognostic indicator in esophageal cancer. Hepatogastroenterology 55: 1503-1508, 2008.

20. Chen M, Cai E, Huang J, Yu P and Li K: Prognostic value of vascular endothelial growth factor expression in patients with esophageal cancer: A systematic review and meta-analysis. Cancer Epidemiol Biomarkers Prev 21: 1126-1134, 2012.

21. Kim KJ, Li B, Winer J, Armanini M, Gillett N, Phillips HS and Ferrara N: Inhibition of vascular endothelial growth factor-induced angiogenesis suppresses tumour growth in vivo. Nature 362: 841-844, 1993.

22. Banneau G, Guedj M, MacGrogan G, de Mascarel I, Velasco V, Schiappa R, Bonadona V, David A, Dugast C, Gilbert-Dussardier B, et al: Molecular apocrine differentiation is a common feature of breast cancer in patients with germline PTEN mutations. Breast Cancer Res 12: R63, 2010.

23. Haier J and Nicolson GL: PTEN regulates tumor cell adhesion of colon carcinoma cells under dynamic conditions of fluid flow. Oncogene 21: 1450-1460, 2002.

24. Zheng HC, Li YL, Sun JM, Yang XF, Li XH, Jiang WG, Zhang YC and Xin Y: Growth, invasion, metastasis, differentiation, angiogenesis and apoptosis of gastric cancer regulated by expression of PTEN encoding products. World J Gastroenterol 9: 1662-1666, 2003.

25. Lu TL, Huang YF, You LR, Chao NC, Su FY, Chang JL and Chen CM: Conditionally ablated Pten in prostate basal cells promotes basal-to-luminal differentiation and causes invasive prostate cancer in mice. Am J Pathol 182: 975-991, 2013.

26. Pan QF, Li WT, Dong HC, Chen YZ, Yin L, Liu W, Wang WW, Liu D, Li SG, Gu WY, et al: PTEN hypermethylation profiles of Chinese Kazakh patients with esophageal squamous cell carcinoma. Dis Esophagus 27: 396-402, 2014.

27. Jiang BH, Zheng JZ, Aoki M and Vogt PK: Phosphatidylinositol 3-kinase signaling mediates angiogenesis and expression of vascular endothelial growth factor in endothelial cells. Proc Natl Acad Sci USA 97: 1749-1753, 2000.

28. Vanhaesebroeck B, Stephens L and Hawkins P: PI3K signalling: The path to discovery and understanding. Nat Rev Mol Cell Biol 13: 195-203, 2012.

29. Zhou YJ, Xiong YX, Wu XT, Shi D, Fan W, Zhou T, Li YC and Huang X: Inactivation of PTEN is associated with increased angiogenesis and VEGF overexpression in gastric cancer. World J Gastroenterol 10: 3225-3229, 2004. 